\title{
Myxedema Coma as Initial Clinical Presentation of Empty Sella Syndrome
}

\author{
Vinoth Kumar Sethuraman, Kavitha B \\ Department of General Medicine, Indira Gandhi Medical College \& Research Institute, Puducherry, India.
}

\author{
Corresponding Author: \\ Dr. Vinoth Kumar Sethuraman \\ Email: kumarsv02@yahoo.com
}

This is an Open Access article distributed under the terms of the Creative Commons Attribution License (creativecommons.org/ licenses/by/3.0).

Received

Accepted

Published

February 16, 2018

September 17, 2018

September 30, 2018

\begin{abstract}
Background: Myxedema coma is a medical emergency seen in patients with primary hypothyroidism. The prognosis is poor if not diagnosed and treated early. Here we report a case of secondary hypothyroidism due to empty sella syndrome presenting with initial manifestation of myxedema coma which is exceedingly rare. Case Report: A middle aged lady presented with easy fatigability for four months, giddiness for one month and vomiting for four days. Clinical diagnosis of myxedema coma was made; she was treated with levothyroxine and laboratory evaluation revealed hypo-pituitarism due to empty sella syndrome. Conclusion: Myxedema coma is an endocrine emergency seen rarely in patients with secondary hypothyroidism but early detection and management reduces the mortality.
\end{abstract}

Keywords: Coma, Empty Sella Syndrome, Hypothyroidism, Myxedema, Thyroxine.

\section{Introduction}

Myxedema coma, the extreme manifestation of hypothyroidism, is commonly seen in patients with primary hypothyroidism. Empty sella syndrome (ESS), is a condition in which the sella turcica is partially or completely filled with cerebrospinal fluid (CSF) resulting in displacement or flattening of the normal pituitary gland, as described by Busch in 1951 [1]. Empty sella is reported to occur in $5.5-23.5 \%$ of the population [2]. Endocrine abnormalities like pan-hypopituitarism, gonadotropin deficiency or diabetes insipidus is seen in about 25-50\% of patients [2]. Myxedema coma is a rare, often fatal endocrine emergency seen in elderly patients with long-standing primary hypo-thyroidism [5]. Myxedema coma of central origin is exceedingly rare [6]. Here we report a case of empty sella syndrome presenting as myxedema coma as the initial clinical manifestation.

\section{Case Report}

A 35 year old female, mother of two children, with last childbirth 15 years back was admitted with complaints of easy fatigability for four months, postural giddiness for one month and vomiting for four days. She had attained menopause four years back and there is no history suggestive of post-partum hemorrhage and lactational failure. On admission patient was drowsy, dehydrated with slowness of speech, cold extremities with coarse dry skin [Fig.1], deep tendon reflexes showed delayed relaxation, hypotension with blood pressure of 90/60 $\mathrm{mm} \mathrm{Hg}$ and capillary blood glucose of $55 \mathrm{mg} / \mathrm{dL}$. In the emergency ward patient was treated with intravenous dextrose and bolus of normal saline. But patient was persistently hypotensive, based on history and clinical findings myxedema coma was suspected. After taking blood samples she was started on intravenous hydrocortisone followed by loading dose of levothyroxine 500 micrograms through Ryles tube along with $25 \%$ dextrose, intravenous antibiotics and normal saline.

Initial laboratory investigations revealed TSH level $2.76 \mu \mathrm{IU} / \mathrm{mL}(0.35-6.16)$, free T3 0.98 $\mathrm{pg} / \mathrm{mL}(1.40-4.20)$ and free T4 $0.42 \mathrm{ng} / \mathrm{dL}(0.80-$ 
2.00) suggestive of secondary hypothyroidism. Complete blood count showed normocytic normochromic anemia. Biochemical analysis showed hyponatremia, elevated creatinine phosphokinase levels with normal renal and liver function tests. Electocardiogram showed sinus bradycardia with low voltage complexes and echocardiogram was normal.

In view of secondary hypothyroidism, computed tomography of brain and other hormone analysis were performed. Computed tomography imaging revealed an empty sella [Fig.2] and hormonal assays showed serum prolactin 2.42 $\mathrm{ng} / \mathrm{mL}$ (4.79-23.2), serum cortisol $4.28 \mu \mathrm{g} / \mathrm{dL}$ (5-25), LH 3.76 mIU/mL(7.7-58.5), FSH $8.18 \mathrm{mIU} /$ $\mathrm{mL}$ (25.8-134.8) suggestive of hypo-pituitarism. MRI brain and testing for growth hormone, ACTH and anti-diuretic hormone were unavailable in our institution. Hyponatremia was corrected with $3 \%$ saline and 100 microgram levothyroxine was continued daily through Ryles tube. Patient became normotensive and mentation improved after 24 hours of admission. After hemodynamic stabilization patient was discharged on levothyroxine 100 microgram and low dose oral prednisolone in view of hypopituitarism. On follow up patient was active and cheerful and free T4 normalized after eight weeks and advised to continue levothyroxine and steroid supplementation.

\section{Discussion}

Empty sella syndrome (ESS) is pathophysiologically characterised by either anatomic abnormalities in the diaphragma sellae (primary ESS) or damage to the pituitary by irradiation, surgery or autoimmunity leading to availability of "empty" space in the sella (secondary ESS) [1]. Empty sella has female preponderance, and primary ESS had been described commonly in middleaged obese women [4]. Empty sella is generally asymptomatic and may be incidentally detected during neuroimaging. In a study by Bianconcini $\mathrm{G}$ et al. in primary empty sella, endocrinopathies

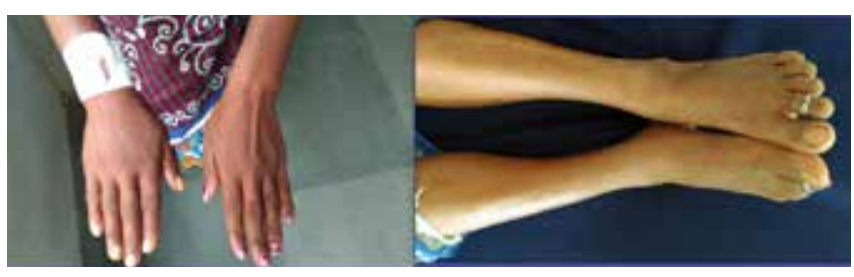

Fig.1: Showing extremities with coarse dry skin.

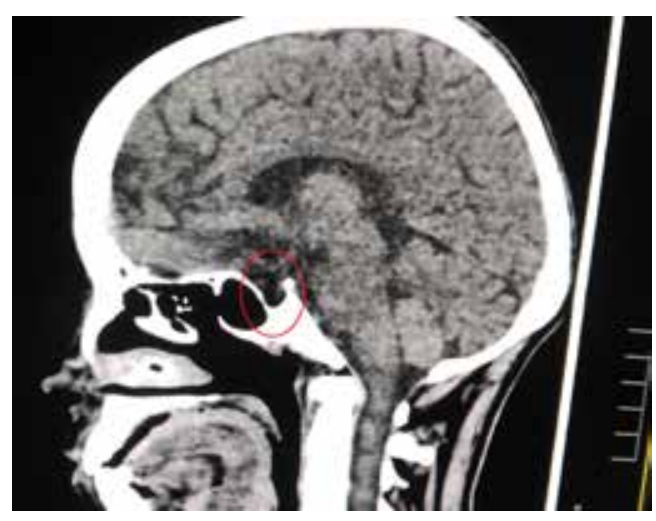

Fig.2: Computed tomography brain showing empty sella.

was seen in 36/71 (50.7\%), isolated or coexisting in some patients: hyper-prolactinaemia (14\%), hypopituitarism (10.4\%), hypo-gonadism (7\%), diabetes insipidus $(2.8 \%)$, hyper-adrenocorticotrophic hormone (1.4\%), hypo-growth hormone (15.4\%), pituitary adenomas (8.4\%) [7]. Neurological dysfunction such as headache and visual abnormalities and endocrine dysfunction related to prolactin, glucose metabolism, gonads, thyroid glands and posterior pituitary are observed $[8,9]$.

Myxedema coma, the extreme manifestation of hypothyroidism is an uncommon but potentially lethal condition [5]. Myxedema coma occurs almost exclusively in elderly with $80 \%$ of the cases occurring in women [10]. Acommon misconception is that a patient must be comatose to be diagnosed with myxedema coma. However, myxedema coma is a misnomer because most patients exhibit neither the non-pitting edema known as myxedema nor coma. Instead, the hallmark presentation of myxedema coma is decreased mental status, along with classic myxedematous face, coarse dry skin, bradycardia and delayed relaxation of deep tendon 
reflexes. Associated features include hypercapnia, hypotension, hypothermia and hypoglycaemia. It is usually precipitated by infections, heart failure, myocardial infarction, stroke, gastrointestinal bleeding and sedatives [11]. Even with early detection and appropriate treatment, mortality ranges from 30 to 60 percent [12].

The association of hypo-thyroidism and hypo-pituitarism of central origin is extremely infrequent [13]. In secondary or tertiary hypothyroidism caused by pituitary or hypothalamic dysfunction respectively, TSH will not be elevated. The diagnosis of secondary or tertiary hypothyroidism is critical from a mortality and management standpoint. An isolated pituitary trophic hormone defect is rare, and most cases of secondary hypothyroidism present with hypopituitarism [6]. In either case of secondary or tertiary hypothyroidism, the TSH and T4 levels are low, as are the gonadotropins in the postmenopausal woman and serum cortisol in both female and male patients.

Treatment of myxedema coma includes intravenous levothyroxine, concurrent therapy with hydrocortisone in view of impaired adrenal reserve and treatment of precipitating illness. Common complications include dilutional hyponatremia, circulatory collapse and respiratory failure [14].

\section{Conclusion}

Empty sella is generally asymptomatic and incidentally detected during imaging but may be accompanied by neurological, endocrine and ophthalmological abnormalities [3]. Myxedema coma may present with the classic myxedema facies, however, not all patients exhibit the full list of classical findings and coma is not required for diagnosis. The most important elements in treatment of myxedema coma are early recognition, presumptive thyroid hormone replacement and appropriate supportive care since it carries a significant mortality even with appropriate testing and treatment.
Contributors: VKS: patient management, manuscript writing, literature review; KB: patient management, critical inputs in the manuscript. VKS will act as guarantor. Both authors approved the final version of this manuscript.

Funding: None; Competing interests: None stated.

\section{References}

1. Sachdev Y, Evered DC, Appleby A, Hall R. The empty sella syndrome. Postgrad Med J. 1976;52:703-705.

2. Jordan RM, Kendall JW, Kerber CW. The primary empty sella syndrome: analysis of the clinical characteristics, radiographic features, pituitary function and cerebrospinal fluid adenohypophysial hormone concentrations. Am J Med. 1977;62:569-580.

3. Aruna P, Sowjanya B, Reddy PA, Krishnamma M, Naidu JN. Partial empty sella syndrome: a case report and review. Ind J Clin Biochem. 2014;29:253-256.

4. Bensing S, Rorsman F, Crock P, Sanjeevi C, Ericson $\mathrm{K}$, Kämpe O, Brismar K, Hulting AL. No evidence for autoimmunity as a major cause of the empty sella syndrome. Exp Clin Endocrinol Diab. 2004;112:231-235.

5. Wall CR. Myxedema coma: diagnosis and treatment. Am Fam Physician. 2000;62:2485-2490.

6. Benvenga S, Squadrito S, Saporito F, Cimino A, Arrigo F, Trimarchi F Myxedema coma of both primary and secondary origin, with non-classic presentation and extremely elevated creatine kinase. Horm Metab Res. 2000;32:364-366.

7. Bianconcini G, Bragagni G, Bianconcini M. Primary empty sella syndrome. Observations on 71 cases. Recenti Prog Med. 1999;90:73-80.

8. Brismar K, Efendić S. Pituitary function in the empty sella syndrome Neuroendocrinology. 1981;32:70-77.

9. Jordan RM, Kendall JW, Kerber CW. The primary empty sella syndrome: analysis of the clinical characteristics, radiographic features, pituitary function and cerebrospinal fluid adenohypophysial hormone concentrations. Am J Med. 1977;62:569-580.

10. Vnthia G, Olsen M. Myxedema coma in the elderly. J Am Board Fam Pract. 1995;8:376-383.

11. Gupta KJ. Myxedema coma: a sleeping giant in clinical practice. Am J Med. 2013;126:e3-4.

12. Gish DS, Loynd RT, Melnick S, Nazir S. Myxoedema coma: a forgotten presentation of extreme hypothyroidism. BMJ Case Rep. Published online doi:10.1136/bcr-2016-216225.

13. Serpico PC, Freeman JS, Marks B. Empty sella syndrome associated with partial hypopituitarism (visualized on MRI scan). J Am Osteopath Assoc. 2007;92:1172-1174.

14. Incasa E, Tampieri M, Zangirolami A, Gamberini S, Di Chiara V, Boari B, et al. Hypothermia with loss of consciousness and hyponatraemia. Intern Emerg Med. 2007;2:113-115. 